\title{
Structure Design of Fiber Bragg Grating Strain Sensor with Built-in Temperature Compensation Grating
}

\author{
Fanyong Meng ${ }^{1, a}$, Limin Jia ${ }^{1, b}$, Xiaohuan Shen ${ }^{2, c}$, Junwei Dong $^{2, d}$ \\ ${ }^{1}$ Beijing Jiaotong University, Research Center of Urban Traffic Information Intelligent Sensing and \\ Serveice Technologies, Beijing China, 100044; \\ ${ }^{2}$ Beijing Pi-optics Co., Ltd, Beijing China, 100176 \\ a35257625@qq.com, 'Imjia@bjtu.edu.cn, ${ }^{\text {c8 } 821499844 @ q q . c o m,{ }^{c} 419059657 @ q q . c o m}$
}

Keywords: optical fiber Bragg grating, sensitivity, temperature compensation, compensation accuracy, the reference grating

\begin{abstract}
Fiber Bragg grating is sensitive to temperature and strain, which lead to the temperature compensation must be considered when the FBG strain sensor is applied. Discussed several typical temperature compensation scheme for the applicability of the FBG strain sensor, analyzed the important factors which affected the compensation accuracy of FBG strain sensor. Based on the reference grating temperature compensation scheme, we designed an encapsulation structure of FBG strain sensor with built-in temperature compensation grating. This structure is simple, practical; also calibration test and outdoor test have proved that it has a good temperature compensation effect.
\end{abstract}

\section{Introduction}

Optical fiber Bragg grating (hereinafter referred to as the grating or FBG) is sensitive to temperature and strain, people use this feature to encapsulate it into temperature sensor, strain sensor and any other series of sensors whose parameters based on temperature or strain. These sensors have been widely used with its characteristics such as light weight and small body, no need power supply, long signal transmission distance, electromagnetic resistent, can be tested by a variety of parameters, easy to monitored by network.

However, in practical application, FBG will brings a lot of troubles to the demodulation of temperature and strain because of its features that sensitive to temperature and strain. To solve this problem, a variety of temperature compensation plans are put forward. But for extensive applied FBG strain sensor, some schemes are not suitable; some applicable schemes affect the effects of temperature compensation by improper use. In order to improve the temperature compensation accuracy of FBG strain sensor, it is necessary to analyses the applicability of the temperature compensation scheme and other factors that affecting the precision of temperature compensation in the process of FBG strain sensor design.

\section{Analyze related problem about temperature compensation of FBG strain sensor}

According to the mechanism of temperature and stress on the role of FBG, the quantitative relationship between Bragg wavelength change, strain variation and temperature variation can be expressed as ${ }^{\mathbf{1 1}}$ :

$$
\frac{\Delta \lambda_{B}}{\lambda_{B}}=\left(1-P_{e}\right) \Delta \varepsilon+(a+\zeta) \Delta T
$$

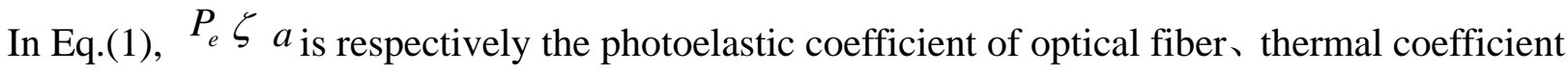
of light 、 thermal expansion coefficient. $\Delta \varepsilon \Delta T$ is respectively variation of strain and temperature.

From Eq.(1) it can be saw intuitively that when only one quantity such as temperature or strain changes, $\Delta \varepsilon_{\text {or }} \Delta T$ is easy to calculation. When both two quantities change, it is unable to directly calculate the value of $\Delta \varepsilon$ or $\Delta T$. In practical application, the temperature and strain exist 
simultaneously, especially in the FBG strain test, constant temperature is almost impossible, so temperature compensation measures must be taken.

The temperature compensation accuracy of FBG strain sensor is influenced by the temperature compensation scheme and technical detail, therefore this section analyses and discusses these problems.

\section{Temperature compensation schemes of FBG strain sensor}

People research on FBG temperature compensation scheme, and put forward a variety of solutions also. The author analyzed and discussed the advantages and disadvantages of these solutions and the applicability of FBG strain sensor, in order to select an optimal temperature compensation scheme.

\section{1)Temperature compensation scheme which use Negative thermal expansion coefficient materials packaging ${ }^{[2]}$}

FBG is fixed to a packaging material which has a negative thermal expansion coefficient, heat shrinkage of Packaging material leads to generate negative axial strain in optical fiber. Using Bragg wavelength negative deviation which is caused by negative strain, offset Bragg wavelength positive deviation which is caused by temperature rise. It is the basic idea of temperature compensation scheme.

The biggest advantage of this scheme is to use a single material, no special structural design, and simple implementation. The disadvantage is difficult to find proper packaging material which has negative thermal expansion coefficient. Besides, FBG strain sensor is fixed closely together to the structure to be tested, which bound free expansion and contraction of encapsulation material. This will not play its normal role. Therefore, this scheme cannot be used for temperature compensation of FBG strain sensor.

\section{2)Temperature compensation by double metals ${ }^{\text {[31 }}$}

This method is to use the thermal expansion property of materials to offset the positive deviation of FBG Bragg grating wavelength along with temperature rise, the structure design as shown in fig.1.

The scheme adapts two materials A and B which have different thermal expansion coefficient. FBG is fixed between A and B. When temperature rises, L1、 L2 have elongation trend. But for the role of FBG, one is a stretch, the other is compressed, and the two actions might offset each other. By selecting the thermal expansion coefficient of material A and B, control the size of the L1 and L2, eventually make the L3 shorter when temperature rise to offset the Bragg wavelength positive deviation.

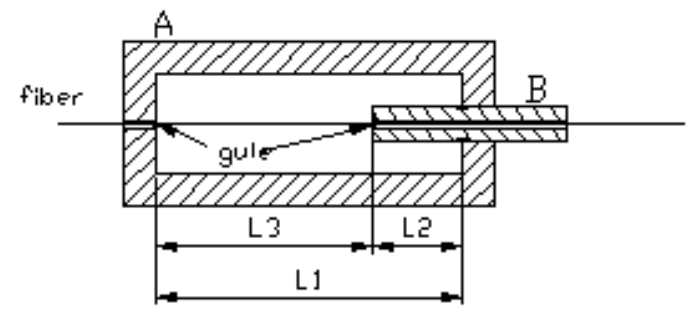

Fig.1. Temperature compensation by double metals

Compared with the scheme which uses negative thermal expansion coefficient material, this scheme adds a size adjustment. It is conducive to easing demand for thermal expansion coefficient to make the design more flexible. But this method has the same disadvantage. After FBG strain sensor is fixed, thermal expansion of Material A will be bound by structure to be tested. If the thermal expansion coefficient of the two is consistent, the scheme is still available, but when the difference is large, it will seriously affect the temperature compensation effect.

So there are a lot of limitations. According to the expansion coefficient of structure, need to adjustment the material and structure to realize temperature compensation.

\section{3)Concatenate double FBG of different diameter}

Weld double FBG of different diameter together, as shown in fig. 2. As the material, the thermal optical coefficient and thermal expansion coefficient is same, two FBG temperature response is 
consistent. The difference of two FBG diameter leads to different strain under the same force.

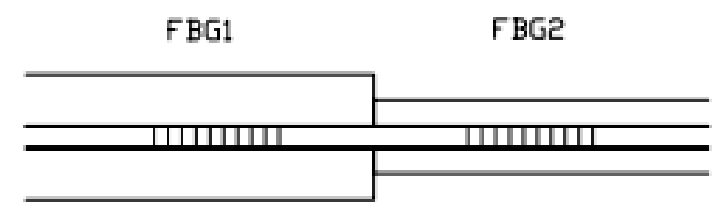

Fig.2. Concatenate double FBG of different diameter

The advantage of this scheme is to calculate the temperature and strain values at the same time; the disadvantage is that the grating concatenated preparation is difficult. If we connect two FBG of different diameter by melding, melting point will be a brittle weak position, affecting the durability. If we change FBG diameter by corroding, process control has certain difficulty. Also measure the diameter accurately is difficult.shi

Therefore, the engineering application of this temperature compensation scheme is difficultly realized.

\section{4)Double grating superposition method}

The double grating superposition method is to write two different Fiber Bragg grating at the same position. Demodulate temperature and strain simultaneously by using sensitivity difference of temperature and strain.

$$
\left[\begin{array}{l}
\Delta \lambda_{B 1} \\
\Delta \lambda_{\mathrm{B} 2}
\end{array}\right]=\left[\begin{array}{ll}
K_{\varepsilon 1} & K_{T 1} \\
K_{\varepsilon 2} & K_{T 2}
\end{array}\right] \bullet\left[\begin{array}{l}
\Delta \varepsilon \\
\Delta T
\end{array}\right]
$$

In Eq.(2), $K_{\varepsilon} 、 K_{T}$ is respectively strain sensitivity coefficient and temperature sensitivity coefficient of FBG wavelength change. Foot mark is used to distinguish between two different wavelengths of FBG. This advantage is simple structure, easy to perpetrate overlapping gratings, realizable to demodulate temperature and strain at the same time. But only when the difference of two FBG wavelengths is big enough, the sensitive coefficient of temperature and strain will have obvious difference, Eq. (2) is able to be solved smoothly. Generally the difference between temperature and strain sensitivity coefficient of two grating which work in the same band is very small, even the existing test system is difficult to distinguish such a small difference. In the paper I ${ }^{1}$ use $1551.7 \mathrm{~nm}$ and $1553.0 \mathrm{~nm}$ grating, realize to demodulate strain and temperature simultaneously. It is feasible in theory, but not much practical significance. And when the grating wavelength is large enough to work in two bands, the system will need two sets of light source and detector, it will no doubt increases the cost and complexity of the system.

Therefore, it is not the best-value temperature compensation scheme of FBG strain sensor.

\section{5)Reference grating temperature compensation scheme ${ }^{\text {71 }}$}

Use a reference FBG which is not sensitive to strain to measure temperature variation $\Delta T$, providing reference temperature for the other FBG which is sensitive to temperature and strain. Although this method requires two FBG, increases the bandwidth utilization, but because of its simple implementation, strong commonality, good compensation effect will be obtained if applied properly.

To take maximum advantages of this temperature compensation scheme, analysis about related problems which affect the accuracy of temperature compensation, in order to provide the reference for structure design of FBG strain sensor which based on this temperature compensation scheme.

\section{Analyze factors which affect the accuracy of temperature compensation of FBG strain sensor}

In practical application, reference grating temperature compensation scheme is often to install a FBG temperature sensor near the FBG strain sensor, as shown in fig.3.

In fig.3, the above is FBG strain sensor; the underside is FBG temperature sensor. This temperature compensation effect in the practical application is often not ideal, especially in the outdoor environment of the test project. The calculated strain results are often affected hugely by the temperature, beyond the scope of reasonable. FBG temperature sensor and FBG strain sensor are placed on the roof, the position is shown as in fig.3, and sensors are free. From November 21, 
2014, at 3pm to November 24, 2014 at 9am, the measured temperature value and strain value which is obtained after temperature compensation is shown in fig.4.

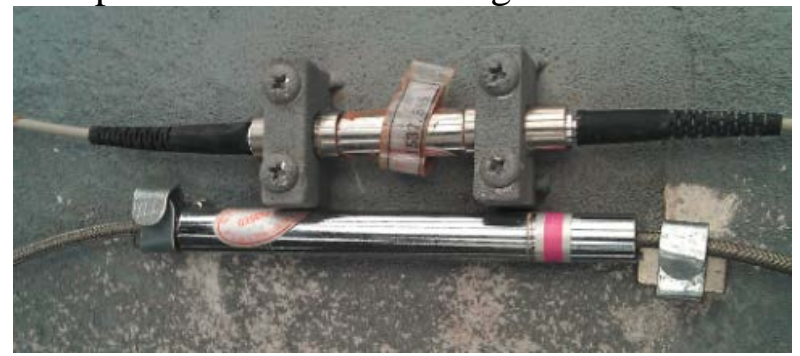

Fig.3. Installation of FBG strain and temperature compensation sensor

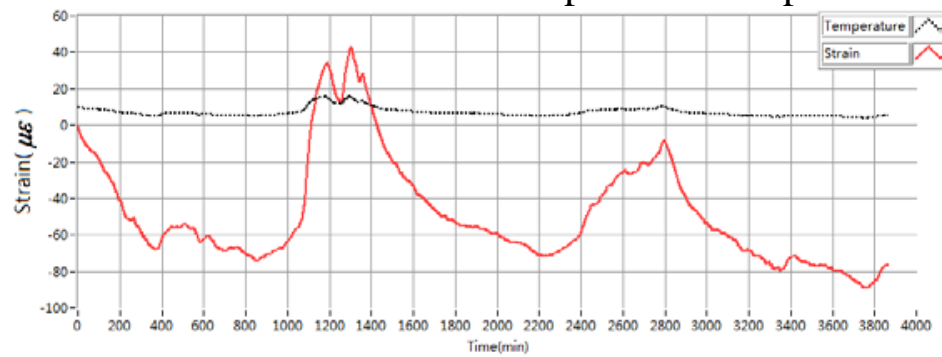

Fig.4. wavelength changes rule of FBG sensor along with temperature changes

Due to sensors which are free, the strain of FBG strain sensor is caused by base material axial linear expansion. According to the compensation for the thermal expansion coefficient of the encapsulation material, the strain value should be close to zero, however in figure 4, the deviation is not only not close to zero but also amplitude is very large.

The author thinks that the main cause of the problem is the temperature compensation accuracy.

For bare grating which work in the 1550nm band, the temperature and strain sensitivity of grating wavelength variation is about $10.8 \mathrm{pm} /{ }^{\circ} \mathrm{C}$ and $1.2 \mathrm{pm} / \mu \varepsilon$. Visible, Compensation temperature appears 1 error may cause nearly $10{ }^{\circ} \mathrm{C}$ of the strain error, evidently the accuracy of the temperature obviously influence on the accuracy of temperature compensation.

The accuracy of temperature are shown in two aspects: on the one hand is the test precision of FBG temperature sensor, and on the other hand is temperature uniformity of FBG temperature sensor and strain sensor. Problem of FBG temperature sensor test precision is easy to understand, and also got the attention of the people. Various measures for increasing accuracy of FBG temperature sensor test has been put forward. In fact, the error due to the inconsistency of temperature between the two FBG sensors is also very obvious, but is often ignored. Fig.5 is the temperature test data, the FBG temperature and strain sensors were handled in accordance with the temperature sensor at noon.

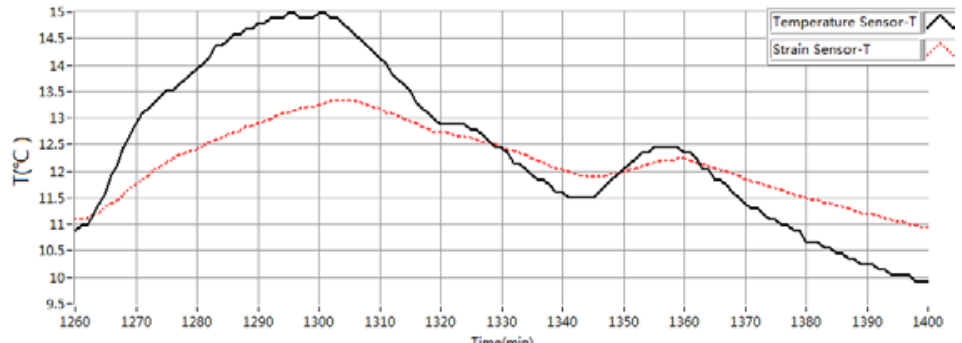

Fig.5. the temperature test data of FBG temperature and strain sensor

Fig. 5 shows that the highest temperature of two sensors are obviously different, the deviation is about $1.5{ }^{\circ} \mathrm{C}$. The speed up and down of temperature between the two is inconsistent; the temperature response speed of FBG strain sensor is obviously lags behind that of FBG temperature sensor. It must lead to obvious strain calculation error if we make temperature compensation by using the temperature at the same moment.

The author believes that the cause of inconsistent on temperature response speed is different forms of packaging, structure, material, and volume. The first heat capacity is different; the second 
transfer efficiency of temperature from inner to outer is different also, eventually led to the difference of temperature response.

In order to solve the temperature test accuracy, this paper proposes an encapsulation structure of FBG strain sensor which can realize integration encapsulation of temperature and strain FBG. As shown in fig. 6 in order to avoid temperature test grating to be affected by packaging substrate deformation, do the bending processing. Compared to the implementation way as shown in fig.3, two FBG are packaged in the same internal structure, which is conductive to improve the temperature consistency. But when the temperature changes fast, it still can appear the response differences.

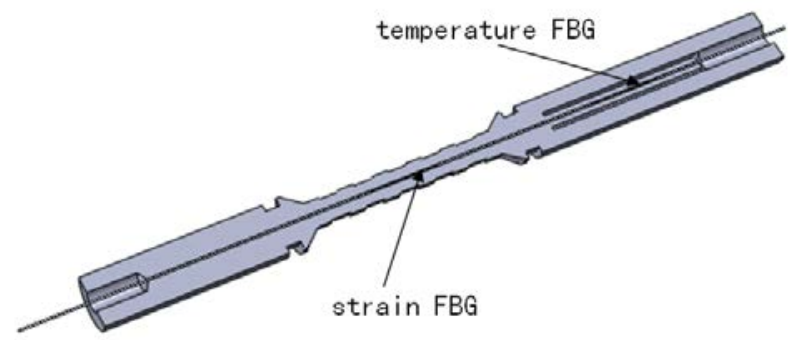

Fig.6. An integration encapsulation of temperature and strain FBG

Encapsulate structure similar shown in fig.6, put the sensor in the temperature-box which has been heated to a temperature of $60{ }^{\circ} \mathrm{C}$ and stable, after 20 minutes, natural cooling. It is concluded that the temperature of the curve as shown in fig.7.

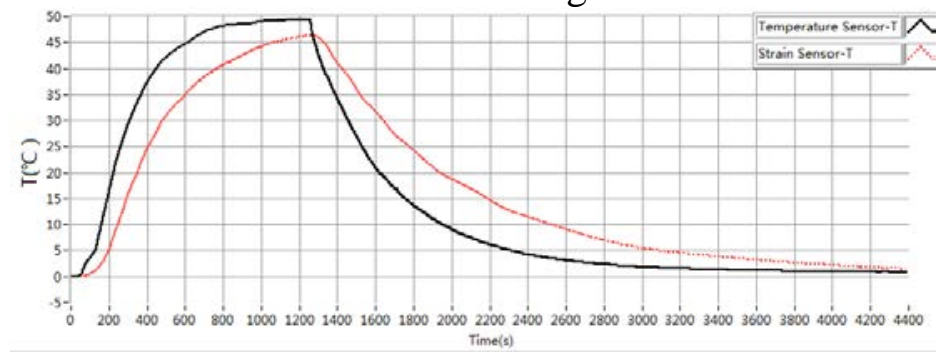

Fig.7. The contrast temperature response when temperature changes rapidly

The fig.7 shows that after 20 minutes the test result of temperature grating is up to $50^{\circ} \mathrm{C}$ and has been basically stable, however, the test temperature of strain grating is still rising. The rising and falling speed of FBG strain test grating is obviously lagging behind the FBG temperature test grating. It obviously still causes error compensation.

The author thinks that the reason which causes the inconsistent temperature response of two FBG is that the states of two grating still have differences. When temperature conduct from outside to inside, the temperature test grating is in a suspended state, heat capacity is small, it can be directly heated. The strain test grating is bonded together with substrate, heat capacity is lager, the heating speed id slow. So it led to the difference.

\section{The encapsulation structure design of FBG strain sensor}

In order to improve the effect of $r$ temperature compensation of FBG strain sensor, improve the strain test accuracy, the author made structure improvement based on the literature of 8 , design an encapsulation structure of FBG strain sensor with a built-in temperature compensation grating.

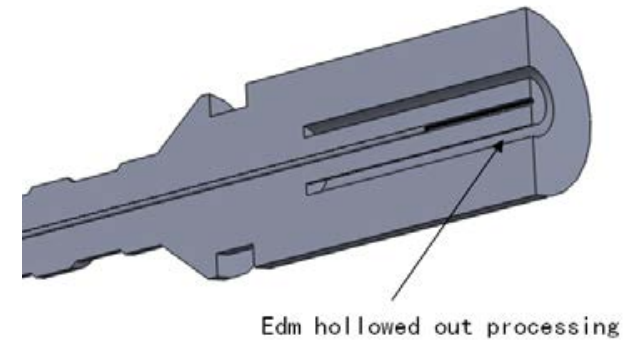

Fig. 8 Sensor encapsulation structure 
Temperature test grating and strain test grating are pasted with the same pull force on the substrate material using 353ND glue. So temperature and strain grating received the similar influence of glue and substrate. It is conducive to improve the temperature response consistency. Due to temperature test grating's position which separated from the substrate, the matrix overall deformation which is caused by the external force will not affect the temperature test grating.

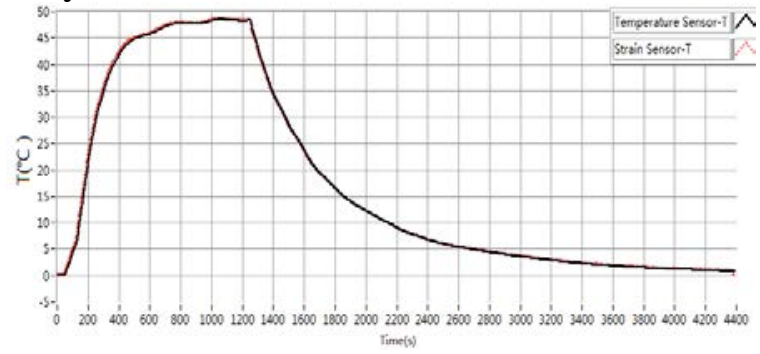

Fig.9. Contrast temperature response curve of two FBG in sensor which is shown as fig.8

Fig.9 is contrast temperature response curve of two grating, two grating's temperature test results are basically identical. So it is helpful to improve the accuracy of temperature compensation.

\section{Conclusions}

Discussed several typical FBG temperature compensation schemes, analyzed the advantages and defects in the applications of FBG strain sensor with temperature compensation. Final selected reference grating temperature compensation scheme as design plan, analyzed that the main causes which lead to not obtain ideal temperature compensation effect in the design scheme is temperature inconsistency. Aimed at the root of the problems, designed a temperature and strain grating integration encapsulated structure of FBG strain sensor, improved the two grating temperature response consistency and the precision of temperature compensation of FBG strain sensor.

\section{Acknowledgements}

This work was supported by the National 863 Plan (2011AA11A102).

\section{References}

[1]Chuan Li, Yimo Zhang, Yonggui Zhao, etc. The principle, technology and sensing application of fiber Bragg grating. Science press, 2005

[2]Yan Wang, Weiping Yan, Yushu Zhang, etc. Research on optical fiber Bragg grating temperature compensation based on negative expansion materials, 2006, vol27, No. 6, p. 10,11,25

[3]Jiping Hu, Naiguang Lv. Research on the Bragg grating temperature compensation based on double metal structure. Electronic test,2008,No.3, P.18-20,27

[4]Guoli Li, Zhiquan Li. Temperature compensation of fiber Bragg grating strain sensor in monitoring. Laser and optoelectronics progress, 2005, Vol. 42, No. 4, P. 25-28

[5]Xu M G, Archambault J L, Reekie L, et al. Discrimination between strain and temperature effects using dual-wave-length fiber grating sensors [J]. Electric Lett,1994, 30, P.1085 1087

[6]Jilong Bao, Xianmin Zhang, Kangsheng Chen. Optical fiber Bragg grating used for temperature and train measurement. ournal of ningbo university (institute), 2003, Vol16 No.1, P.49-52

[7]Libing Wan, Dianfu Wang. Temperature compensation of fiber Bragg grating strain sensor based on reference grating. Photoelectron forced the laser, 2006, Vol17, No. 1, P. 50-53

[8]Zhenbao Wang,Pengling Yang, Bibo Shao, etc. Double parameters stress and temperature measurement fiber Bragg grating sensor Laser and optoelectronics progress. 2013, 50, 100602, 1-5 